\title{
Existence of the Spontaneous Pair Creation in the External Field Approximation of Q.E.D.
}

\author{
G. Nenciu * \\ Max-Planck-Institut für Physik und Astrophysik, Werner Heisenberg Institut für Physik, \\ Föhringer Ring 6, D-8000 München 40, Federal Republic of Germany
}

\begin{abstract}
The problem of the spontaneous pair creation in strong electromagnetic fields is considered in the adiabatic switching formalism. The existence of a critical value of the strength of the external field at which the behaviour of the pair production probability has an abrupt change is proved.
\end{abstract}

\section{Introduction}

This note is a direct continuation of [1], and concerns the existence of the overcritical fields in the framework of the external field approximation of Q.E.D. (see [2] and references therein). In [1] the existence of the overcritical fields (or in other words the existence of the spontaneous pair creation) was viewed as the breakdown of the adiabatic switching theorem: for sufficiently strong external fields the pair creation is by far more copious than one expected from the time variation of the external fields; in particular it does not vanish as the "speed" of the time variation of the external fields goes to zero. This is completely different from the low field regime where the pair creation comes entirely from the time variation of the external fields and vanishes in the adiabatic limit, i.e. the adiabatic limit coincides with the static result [3]. It is believed (for references to the physical literature about the subject see [4-6]) that the change in the behavior of the pair production probability is an abrupt one and takes place at a value of the external field called the critical field.

In [1] the notions of the "undercritical" and "overcritical" static fields were made precise, some conjectures were put forward and the "undercritical" part of these conjectures was proved. On the other hand, the important problem of the existence of the overcritical fields was left open. Unfortunately, due to a technical difficulty (see the remark at the end of Sect. 3) at the present we are unable to prove the existence of the overcritical fields as described by the conjectures put forward in [1]. However, we are able to prove the existence of the sudden change from

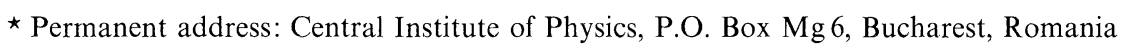


undercritical to overcritical regime in a slightly modified context (which is reasonable from the physical point of view, although less satisfactory from the aesthetic point of view). In spite of the fact that we consider the result in the present note ephemeral (we believe that the more elegant and natural conjecture in [1] is true and someone will prove it) we decided to publish it for the following two reasons: i) the existence of a critical field in the framework of scattering theory has been challenged in the recent literature $[7,8]$; ii) describes a critical phenomenon which is, in our opinion, interesting both from physical and mathematical point of view.

In Sect. 2 the problem is briefly described and the main result stated. Section 3 contains the proof.

\section{Description of the Problem and the Result}

The problem to be discussed is the behaviour of the quantized Dirac field subjected to the influence of a classical external field. The time evolution is governed by the Eq.

$$
i \frac{d}{d t} \psi_{t}(x)=\left(\alpha p+\beta m+\mathbf{V}_{t}(x)\right) \psi_{t}(x) ; x \in \mathbb{R}^{3}
$$

As is well known (see references in [1]) under fairly general conditions on the external field, $\mathbf{V}_{t}(x)$, the scattering theory for (2.1) can be described in terms of the scattering matrix $\mathscr{S}(\mathbf{V})$, where $\mathscr{S}(\mathbf{V})$ is a unitary operator in the Fock-Cook space $\mathscr{F}_{0}$ of the free Dirac field.

In what follows we shall consider only one matrix element of $\mathscr{S}(\mathbf{V})$, which describes one of the most interesting quantities of the theory, namely the probability of pair production from the vacuum state, given by

$$
r(\mathbf{V})=1-|(\Omega, \mathscr{S}(\mathbf{V}) \Omega)|^{2},
$$

where $\Omega$ is the vacuum state in $\mathscr{F}_{0}$. According to the general discussion in $[1,2]$ we shall take $\mathbf{V}_{t}(x)$ of the following form:

$$
\mathbf{V}_{t}(x)=\lambda \varphi_{\delta}(\varepsilon t) \mathbf{V}(x)
$$

where $\lambda \in \mathbb{R}^{+}, \mathbf{V}$ is a nice (see condition B below) $4 \times 4$ hermitian matrix valued function and $\varphi_{\delta}(s)$ is a "switching factor" of a more general type than those considered in [1], namely $\varphi_{\delta}(s)$ is supposed to satisfy the following conditions:

i) $0 \leqq \varphi_{\delta}(s) \leqq 1, \varphi_{\delta}(0)=1, \lim _{s \rightarrow \pm \infty} \varphi_{\delta}(s)=0$,

ii) Outside a finite set $\{I\}, 0 \neq\{I\}, \varphi_{\delta}(s)$ is twice differentiable and

$$
\begin{aligned}
& \sup _{s \in \mathbb{R} \backslash\{I\}}\left|\frac{d}{d s} \varphi_{\delta}(s)\right|<\infty, \lim _{s \rightarrow \pm \infty} \frac{d}{d s} \varphi_{\delta}(s)=0 \\
& \underset{\mathbb{R} \backslash\{I\}}{\int}\left(\left|\frac{d}{d s} \varphi_{\delta}(s)\right|^{2}+\left|\frac{d^{2}}{d s^{2}} \varphi_{\delta}(s)\right|\right) d s<\infty,
\end{aligned}
$$

iii) $\max _{s \in\{I\}}\left|\varphi_{\delta}(s-0)-\varphi_{\delta}(s+0)\right|=\delta$. 
In general, for $\varepsilon, \delta \neq 0, r(\mathbf{V}) \neq 0$. Following the physical literature we shall call $\lambda \mathbf{V}(x)$ undercritical if for small $\delta$, in the adiabatic limit $\varepsilon \rightarrow 0, r(\mathbf{V})$ is also (of order $\delta$ say) small, i.e. only the "normal" pair creation due to the small jumps of the external field at $\varepsilon t \in\{I\}$ is present. If, on the contrary, even for $\varepsilon, \delta \rightarrow 0 r(\mathbf{V}) \simeq 1, \lambda \mathbf{V}$ is called "overcritical." According to the more or less generally accepted belief for a given $\mathbf{V}(x)$ there exists $\lambda_{\text {cr }}$ (see [1] Sect. 2 and also Lemma 1 below) such that $\lambda \mathbf{V}$ is undercritical for $0 \leqq \lambda<\lambda_{\mathrm{cr}}$ and overcritical for $\lambda>\lambda_{\mathrm{cr}}$.

In this note we shall study $r(\mathbf{V})$ in a neighbourhood of $\lambda_{\mathrm{cr}}$ and prove that for an appropriate class of $\mathbf{V}(x)$ and $\varphi_{\delta}(s)$ (see the conditions $\mathrm{A}, \mathrm{B}$ at the end of this section), $r(\mathbf{V})$ indeed has a jump at $\lambda_{\mathrm{cr}}$ in the limit $\varepsilon \rightarrow 0$. More exactly

Proposition 1. There exist $\varphi_{\delta}(s), V(x)$ such that the following is true:

If $\mathbf{V}_{t}(x)=\lambda \varphi_{\delta}(\varepsilon t) V(x) \mathbb{1}$, then there exists $\lambda_{\mathrm{cr}}$ depending on $V(x), \eta(\delta)>0$, $\lim _{\delta \rightarrow 0} \eta(\delta)=0$ such that

$$
\lim _{\varepsilon \rightarrow 0} r(\mathbf{V})\left\{\begin{array}{lll}
\leqq \eta(\delta) & \text { for } & 0 \leqq \lambda<\lambda_{\mathrm{cr}} \\
\geqq 1-\eta(\delta) & \text { for } & \lambda \in\left(\lambda_{\mathrm{cr}}, \lambda_{\mathrm{cr}}+3 \delta / 4 \pi\right) .
\end{array}\right.
$$

Remark. The above result implies in particular that for sufficiently small $\delta, \lim _{\varepsilon \rightarrow 0} r(\mathbf{V})$ has a jump at $\lambda=\lambda_{\text {cr }}$. Let us stress that this discontinuity appears only in the limit $\varepsilon \rightarrow 0$. For fixed (nonzero) $\varepsilon$ one can prove [8] that $(\Omega, \mathscr{S}(\mathbf{V}) \Omega)$ is an entire function of $\lambda$, and then $r(\mathbf{V})$ is a smooth function of $\lambda$. Actually this analyticity together with the fact that $r(\mathbf{V})=0$ for static external fields [3] were the arguments in $[7,8]$ for the claim that the spontaneous pair creation does not occur. In our opinion the situation is analogous with the situation in the theory of phase transitions where the order parameter is identified via a sequence of appropriate limits. Actually the analogy with the theory of phase transitions goes a little bit further. The undercritical regime corresponds to the high temperature region; the weak time variation of $\mathbf{V}_{t}(x)$ due to $\varepsilon \neq 0$ [which breaks the time translation symmetry responsible for the fact that $r(\mathbf{V})=0$ for the static case] corresponds to the small external magnetic field in the theory of ferromagnetism, etc. Of course one has not to push this analogy too far.

We end this section describing $\varphi_{\delta}(s)$ and $V(x)$ appearing in Proposition 1.

(A) The switching factor $\varphi_{\delta}(s)$ has the following additional properties:

iv) $\{I\}=\{-1,1\}$,

v) $\varphi_{\delta}(s)=\left\{\begin{array}{lll}1 & \text { for } & |s|<1 \\ 0 & \text { for } & |s|>2\end{array}\right.$

vi) $\varphi_{\delta}(-s)=\varphi_{\delta}(s)$,

vii) $0<\varphi_{\delta}(s)<1-\delta$ for $1<|s|<2 ; \varphi_{\delta}(-1-0)=\varphi_{\delta}(1+0)=1-\delta$.

(B) Let

$$
V_{R}(x)=\left\{\begin{array}{rl}
-1 & |x|<R \\
0 & |x|>R
\end{array} .\right.
$$

Then $V(x)=V(|x|), V(x) \in C_{0}^{\infty}\left(\mathbb{R}^{3}\right)$ and

$$
V_{1-d}(x) \geqq V(x) \geqq V_{1}(x),
$$

where $d$ is a sufficiently small positive number. 


\section{The Proof}

We need some spectral properties of the Hamiltonian summarized in:

Lemma 1. Let

$$
H_{\lambda}=\alpha p+\beta m+\lambda V(x) ; \lambda \in \mathbb{R}^{+},
$$

where $V(x)$ satisfies $B$. Then $H_{\lambda}$ has the following spectral properties

i) $\sigma_{\text {ess }}\left(H_{\lambda}\right)=(-\infty,-m] \cup[m, \infty)$ and is absolutely continuous.

ii) As $\lambda$ increases from zero, the lowest eigenvalue $E_{g}(\lambda)$ appears at $m$ and disappear at $-m$. Let $\lambda_{\mathrm{cr}}$ be the value of $\lambda$ at which $E_{g}(\lambda)$ reaches $-m$. In a (left) neighbourhood of $\lambda_{\mathrm{cr}}, E_{g}(\lambda)$ is nongenerate and

$$
0>\frac{d}{d \lambda} E_{g}(\lambda) \geqq-\frac{4 \pi}{3} .
$$

iii) Let $P_{g}(\lambda)$ be the spectral projection of $H_{\lambda}$ corresponding to $E_{g}(\lambda)$. Then

$$
\text { norm- } \lim _{\lambda \rightarrow \lambda_{\text {cr }}} P_{g}(\lambda) \equiv P_{g}\left(\lambda_{\text {cr }}\right)
$$

exists. $P_{g}(\lambda)$ is norm continuous on $\left[\lambda_{\mathrm{cr}}-\lambda_{0}, \lambda_{\mathrm{cr}}\right]$ for some $\lambda_{0}>0$.

iv) Let $E \notin \sigma\left(H_{\lambda}\right), \lambda \in\left(\lambda_{1}-\mu, \lambda_{1}+\mu\right)$ and $P_{E}^{ \pm}(\lambda)$ be the spectral projections of $H_{\lambda}$ corresponding to $(-\infty, E)$ and $(E, \infty)$ respectively.

Then $P_{E}^{ \pm}(\lambda)$ are (as functions of $\lambda$ ) Hilbert-Schmidt norm differentiable on $\left(\lambda_{1}-\mu, \lambda_{1}+\mu\right)$.

Proof of Lemma 1. i) Obvious under our conditions on $V$. In particular the absence of the eigenvalues in the continuum follows from the spherical symmetry.

ii) For the potential $\lambda V_{R}$ the eigenfunctions can be expressed in terms of Bessel functions in both regions $r<R$ and $r>R(r=|x|)$ and the continuity condition at $r=R$ leads to a transcendental equation for the eigenvalues (see e.g. $[4,9]$ for details). In particular the values of $\lambda$ at which the eigenvalues reach $-m$ are given by

$$
j_{l}(\sqrt{\lambda(\lambda+2 m} R)=0
$$

where $j_{1}(z), l=0,1, \ldots$ are the spherical Bessel functions [10, Sect. 10].

From the ordering of the zeros of the Bessel functions [10, Sect. 10] it follows that at least in a neighbourhood of $\lambda_{\mathrm{cr}}$ the lowest eigenvalue corresponds to total angular momentum $1 / 2$ and positive parity (i.e. $l=0$ or the $s$-wave). The next remark is that although $H_{0}$ is not bounded from below there is a comparison theorem for the eigenvalues. Let $H(\mu)=\alpha p+\beta m+\mathbf{V}+\mu \mathbf{W}, \mathbf{W}=W \mathbb{1}, W(x)<0$, $\|W\|_{\infty}<\infty$.

If $E_{\mu}$ is a nondegenerate eigenvalue of $H(\mu)$, then by perturbation theory

$$
0>\frac{d}{d \mu} E_{\mu}=\left(\psi_{\mu}, W \psi_{\mu}\right) \geqq-\|W\|_{\infty},
$$

where $\psi_{\mu}$ is the corresponding eigenvector. The strict inequality in (3.3) comes from the fact that $\left\|\psi_{\mu} \chi_{\text {supp } W}\right\|$ cannot be zero. In particular (3.3) implies (3.1) and the fact 
that the lowest eigenvalue corresponding to $\lambda V$ lies between the lowest eigenvalues corresponding to $\lambda V_{1-d}$ and $\lambda V_{1}$ respectively (as far as all exist, i.e. appeared from the upper continuum and have not disappeared in the lower continuum). Since the eigenvalues of $H_{0}+\lambda V_{R}$ depend continuously on $R$, the arguments above prove ii) and the fact that $E_{g}(\lambda)$ corresponds to the $s$-wave.

iii) Due to the spherical symmetry the eigenfunction, corresponding to the lowest bound state, has the form

$$
\psi(x)=\left(\begin{array}{c}
g(r) \chi_{-1}^{\mu}(x / r) \\
i f(r) \chi_{1}^{\mu}(x / r)
\end{array}\right)
$$

(see [4] for the definition of $\chi_{ \pm 1}^{\mu}$ ). The coupled radial equations for the functions $f$ and $g$ are

$$
\begin{gathered}
\left(\frac{d}{d r}+\frac{2}{r}\right) f(r)+\left(E_{g}(\lambda)-m-V(r)\right) g(r)=0, \\
\frac{d}{d r} g(r)-\left(E_{g}(\lambda)+m-V(r)\right) f(r)=0 .
\end{gathered}
$$

The solution of (3.4) square integrable at infinity is, for $r>1$,

$$
\begin{aligned}
& f(r)=-N \beta\left(E_{g}(\lambda)+m\right)^{-1} \exp (-\beta r)\left(1 / \beta r+1 /(\beta r)^{2}\right), \\
& g(r)=N \exp (-\beta r) / \beta r,
\end{aligned}
$$

where $\beta=\left(m^{2}-E_{g}^{2}(\lambda)\right)^{1 / 2}$ and $N$ is the normalization factor determined from the condition

$$
\left\|\psi_{g}(\lambda)\right\|^{2}=1=\int_{0}^{\infty} r^{2}\left(f^{2}(r)+g^{2}(r)\right) d r .
$$

Using (3.5) and the fact that

$$
\int_{1}^{\infty} r^{2}\left(f^{2}(r)+g^{2}(r)\right) d r<1
$$

one can easily show that for $\beta \leqq 1 / 2, r_{0}>1$, there exists a constant $K$ independent of $\beta$ and $r_{0}{ }^{1}$ such that

$$
\int_{r_{0}}^{\infty} r^{2}\left(f^{2}(r)+g^{2}(r)\right) d r \leqq K / r_{0}
$$

This implies that $\psi_{g}(\lambda)$ is uniformly localized as $\lambda$ approaches $\lambda_{\mathrm{c}}$, i.e. for every $\gamma>0$ there exists $r(\gamma)$ independent of $\lambda$ such that

$$
\left\|\psi_{g}(\lambda) \chi_{r(\gamma)}\right\| \geqq 1-\gamma
$$

where $\chi_{r(\gamma)}$ is the characteristic function of the ball $|x|<r(\gamma)$.

Consider now (3.4) on the compact $[0, r(\gamma)]$, with regular boundary conditions at the origin [selected by the square integrability of $\left.\psi_{g}(\lambda)\right]$. Using the continuous dependence on the coefficients of the solutions of differential equations [11,

\footnotetext{
${ }^{1}$ The mass, $m$, is supposed to be fixed. The localisation of $\psi_{g}(\lambda)$ is not uniform in $m$, as $m \rightarrow 0$
} 
Chap. 3] and taking into account (3.1) one can find $\lambda(\gamma)$ such that for all $\lambda(\gamma)<\lambda_{1}, \lambda_{2}<\lambda_{\text {cr }}$

$$
\left\|\psi_{g}\left(\lambda_{1}\right)-\psi_{g}\left(\lambda_{2}\right)\right\| \leqq 3 \gamma
$$

which finishes the proof of iii).

For iv) see $[12,13]$.

Proof of Proposition 1. Let $U_{\varepsilon, \lambda, \delta}\left(t, t_{0}\right)$ be the evolution corresponding to

$$
H_{0}+\mathbf{V}_{t}(x)=\alpha p+\beta m+\lambda \varphi_{\delta}(\varepsilon t) V(x) \text {. }
$$

Taking into account the form of $\varphi_{\delta}(s)$, the corresponding scattering matrix $S_{\varepsilon, \lambda, \delta}$ is given by

$$
S_{\varepsilon, \lambda, \delta}=\exp \left(2 i H_{0} / \varepsilon\right) U_{\varepsilon, \lambda, \delta}(2 / \varepsilon,-2 / \varepsilon) \exp \left(2 i H_{0} / \varepsilon\right)
$$

Let

$$
A=P_{0}^{+}(0) S_{\varepsilon, \lambda, \delta} P_{0}^{-}(0), \quad B=P_{0}^{+}(0) S_{\varepsilon, \lambda, \delta} P_{0}^{+}(0) .
$$

Under our conditions on $V, A$ is Hilbert-Schmidt and the basic result of the quantized theory gives the following formula for $(\Omega, \mathscr{S}(\mathbf{V}) \Omega)$ [14],

$$
(\Omega, \mathscr{S}(\mathbf{V}) \Omega)=\left\{\begin{array}{l}
0 \text { if } A A^{*} \text { has } 1 \text { as one of its eigenvalues } \\
\operatorname{det}\left(1+B^{-1} A A^{*} B^{*-1}\right)^{-1 / 2} \text { otherwise }
\end{array} .\right.
$$

From the unitarity of $S_{\varepsilon, \lambda, \delta}$, it follows that [as operators in $P_{0}^{+}(0) \mathscr{H}$ ],

$$
B B^{*}=1-A A^{*} \text {. }
$$

If $\left\|A A^{*}\right\| \leqq b<1$, then $B$ is invertible in $P_{0}^{+}(0) \mathscr{H}$ and $\left\|\left(B B^{*}\right)^{-1}\right\|<1 /(1-b)$. Suppose now $\|A\|_{\text {H.S. }}=a<1$. Then

$$
\operatorname{Tr} B^{-1} A A^{*} B^{*-1} \leqq a^{2} /(1-a)^{2},
$$

and since for a positive operator $C[15$, Chap. 3]

$$
1>\operatorname{det}(1+C)^{-1 / 2} \geqq(\exp \operatorname{Tr} C)^{-1 / 2},
$$

what we have to prove in the undercritical case is that $\|A\|_{\text {H.S. }}$ is sufficiently small.

Suppose now $\|A\|=c \leqq 1$ [by (3.8)]. If $c=1$, then $A A^{*}$ has 1 as an eigenvalue and $r(\mathbf{V})=1$ by the upper part of (3.7). Suppose $c<1$. Then (recall that $A$ is H.S.) $c^{2}$ is an eigenvalue of $A A^{*}$ and from (3.8), $1-c^{2}$ is an eigenvalue of both $B B^{*}$ and $B^{*} B$. From (3.8) and the invertibility of $B$

$$
1=\left(B^{*} B\right)^{-1}-B^{-1} A A^{*} B^{*-1},
$$

and $c^{2} / 1-c^{2}$ is an eigenvalue of $B^{-1} A A^{*} B^{*-1}$. Obviously

$$
\operatorname{det}\left(1+B^{-1} A A^{*} B^{*-1}\right)^{-1 / 2}<\left(1-c^{2}\right)^{1 / 2} .
$$

Taking into account the lower part of the formula (3.7), it follows that in order to prove the existence of the overcritical regime we have to show that in the limit $\varepsilon \rightarrow 0$, $\left\|P_{0}^{+}(0) S_{\varepsilon, \lambda, \delta} P_{0}^{-}(0)\right\|$ is close to one. 
Consider first the undercritical regime. According to the discussion above, what we need are estimations on $\left\|P_{0}^{+}(0) S_{\varepsilon, \lambda, \delta} P_{0}^{-}(0)\right\|_{\text {H.S. }}$ in the limit $\varepsilon \rightarrow 0$. Since $P_{0}^{ \pm}(0)$ are nothing but the spectral projections of $H_{0}$ corresponding to $[m, \infty)$ and $(-\infty,-m]$ respectively we have

$$
\left\|P_{0}^{+}(0) S_{\varepsilon, \lambda, \delta} P_{0}^{-}(0)\right\|_{\text {H.S. }} \leqq\left\|P_{0}^{+}(0) U_{\varepsilon, \lambda, \delta}(2 / \varepsilon,-2 / \varepsilon) P_{0}^{-}(0)\right\|_{\text {H.S. }} .
$$

Let now $\lambda<\lambda_{\text {cr }}$ and $E=\left(E_{g}(\lambda)-m\right) / 2$. Clearly $E>-m$ and $E \notin \sigma\left(H_{0}+\mathbf{V}_{t}(x)\right)$ for all $t \in \mathbb{R}$ so $P_{E}^{ \pm}\left(\lambda \varphi_{\delta}(\varepsilon t)\right)$ are well defined for all $t$ except $t= \pm 1 / \varepsilon$, where they have a jump. Writing for brevity $P_{E}^{ \pm}\left(\lambda \varphi_{\delta}(\varepsilon t)\right) \equiv P_{E}^{ \pm}(t)$, one has (with a self-explanatory notation)

$$
\begin{aligned}
\| P_{0}^{+} & (2 / \varepsilon) U_{\varepsilon, \lambda, \delta}(2 / \varepsilon,-2 / \varepsilon) P_{0}^{-}(-2 / \varepsilon) \|_{\text {H.S. }} \\
= & \| P_{0}^{+}(2 / \varepsilon) U_{\varepsilon, \lambda, \delta}(2 / \varepsilon, 1 / \varepsilon)\left(P_{E}^{+}(1 / \varepsilon+0)+P_{E}^{-}(1 / \varepsilon+0)\right) \\
& \times U_{\varepsilon, \lambda, \delta}(1 / \varepsilon,-1 / \varepsilon)\left(P_{E}^{+}(-1 / \varepsilon-0)+P_{E}^{-}(-1 / \varepsilon-0)\right) U_{\varepsilon, \lambda, \delta}(-1 / \varepsilon,-2 / \varepsilon) \\
& \times P_{0}^{-}(-2 / \varepsilon)\left\|_{\text {H.S. }} \leqq\right\| P_{0}^{+}(2 / \varepsilon) U_{\varepsilon, \lambda, \delta}(2 / \varepsilon, 1 / \varepsilon) P_{E}^{-}(1 / \varepsilon+0) \|_{\text {H.S. }} \\
& +\left\|P_{E}^{+}(-1 / \varepsilon-0) U_{\varepsilon, \lambda, \delta}(-1 / \varepsilon,-2 / \varepsilon) P_{0}^{-}(-2 / \varepsilon)\right\|_{\text {H.S. }} \\
& +\left\|P_{E}^{+}(1 / \varepsilon+0) U_{\varepsilon, \lambda, \delta}(1 / \varepsilon,-1 / \varepsilon) P_{E}^{-}(-1 / \varepsilon-0)\right\|_{\text {H.S. }} .
\end{aligned}
$$

Following closely the proof of Theorem 4 in [1] the first two terms vanish in the limit $\varepsilon \rightarrow 0$. Taking into account that

$$
\left[U_{\varepsilon, \lambda, \delta}(1 / \varepsilon,-1 / \varepsilon), P_{E}^{ \pm}( \pm 1 / \varepsilon \mp 0)\right]=0
$$

[recall that $\varphi_{\delta}(s)$ is constant on $(-1,1)$ ], one obtains that the third term in the r.h.s. of (3.9) is smaller than $2\left\|P_{E}^{+}(1 / \varepsilon+0)-P_{E}^{+}(1 / \varepsilon-0)\right\|_{\text {H.S. }}$. Now

$$
P_{E}^{+}(1 / \varepsilon \pm 0)=P_{g}(1 / \varepsilon \pm 0)+P_{\tilde{E}}^{+}(1 / \varepsilon \pm 0),
$$

where $\widetilde{E}=\left(E_{1}(\lambda)+E_{g}(\lambda)\right) / 2$, and $E_{1}$ is the next eigenvalue of $H_{0}+\lambda \varphi_{\delta}(1-0) V$ and

$$
\begin{aligned}
& \left\|P_{E}^{+}(1 / \varepsilon+0)-P_{E}^{+}(1 / \varepsilon-0)\right\|_{\text {H.S. }} \leqq\left\|P_{g}(1 / \varepsilon+0)-P_{g}(1 / \varepsilon-0)\right\|_{\text {H.S. }} \\
& \quad+\left\|P_{E}^{+}(1 / \varepsilon+0)-P_{\tilde{E}}^{+}(1 / \varepsilon-0)\right\|_{\text {H.S. }} .
\end{aligned}
$$

Since for all $\lambda \in\left(0, \lambda_{\mathrm{cr}}\right], E_{1}$ remains far away from $-m$, due to Lemma $\left.1 \mathrm{iv}\right)$, there exists a constant independent of $\lambda \in\left(0, \lambda_{\mathrm{cr}}\right]$ such that

$$
\left\|P_{\tilde{E}}^{+}(1 / \varepsilon+0)-P_{\tilde{E}}^{+}(1 / \varepsilon-0)\right\|_{\text {H.s. }} \leqq \text { const } \delta .
$$

On the other hand, since $P_{g}(t)$ is one dimensional, taking into account Lemma 1 iii),

$$
\left\|P_{g}(1 / \varepsilon+0)-P_{g}(1 / \varepsilon-0)\right\|_{\text {H.S. }} \leqq \tilde{\eta}(\delta)
$$

with $\tilde{\eta}(\delta) \rightarrow 0$ as $\delta \rightarrow 0$, uniformly with respect to $\lambda \in\left(\lambda_{\mathrm{cr}}-\lambda_{0}, \lambda_{\mathrm{cr}}\right]$ for some $\lambda_{0}>0$. Summing up it follows that

$$
\lim _{\varepsilon \rightarrow 0}\left\|P_{0}^{+}(0) S_{\varepsilon, \lambda, \delta} P_{0}^{-}(0)\right\|_{\text {H.S. }} \leqq \text { const } \delta+\tilde{\eta}(\delta),
$$

which proves the undercritical part of the proposition. 
Consider now the overcritical case. The problem is to find a vector (depending on $\varepsilon$ ) in $P_{0}^{+}(0) \mathscr{H}$ which in the limit $\varepsilon \rightarrow 0$, is "carried" almost completely into $P_{0}^{-}(0) \mathscr{H}$ by the evolution $U_{\varepsilon, \lambda, \delta}(2 / \varepsilon,-2 / \varepsilon)$. Again we shall write $E_{g}(t), \psi_{g}(t)$ as a shorthand for $E_{g}\left(H_{0}+\lambda \varphi_{\delta}(\varepsilon t) V(x)\right)$ and the corresponding normalized eigenvector. Let $\lambda_{1}$ be the value of $\lambda$ at which $E_{g}(-1 / \varepsilon-0)$ reaches $-m$.

Due to (3.1)

$$
\lambda_{1}-\lambda_{\mathrm{cr}} \geqq \delta / 4 \pi \text {. }
$$

Consider now $\lambda \in\left(\lambda_{\text {cr }}, \lambda_{1}\right)$ and $\psi_{\varepsilon}(t)=U_{\varepsilon, \lambda, \delta}(t,-1 / \varepsilon) \psi_{g}(-1 / \varepsilon-0)$. We claim that $P_{0}^{+}(0) \psi_{\varepsilon}(-2 / \varepsilon)$ has the desired property. By the adiabatic theorem $[16,17]$,

$$
\lim _{\varepsilon \rightarrow 0}\left\|P_{0}^{-}(0) \psi_{\varepsilon}(-2 / \varepsilon)\right\|=0
$$

So that in the limit $\varepsilon \rightarrow 0$ one can identify $P_{0}^{+}(0) \psi_{\varepsilon}(-2 / \varepsilon)$ with $\psi_{\varepsilon}(-2 / \varepsilon)$. Now, for sufficiently small $\delta$ there exists $\tilde{\tilde{E}}>E_{g}(-1 / \varepsilon-0)$ such that $H_{0}+\lambda \varphi_{\delta}(-1+0) V$ has no eigenvalues in $(-m, \tilde{\tilde{E}})$. Then by Lemma 1 iii),

$$
\begin{aligned}
& \left\|P_{-m}^{-}(-1 / \varepsilon+0) \psi_{g}(-1 / \varepsilon-0)\right\|=\left\|P_{\tilde{E}}^{\bar{E}}(-1 / \varepsilon+0) \psi_{g}(-1 / \varepsilon-0)\right\| \\
& \quad \geqq 1-\left\|P_{\tilde{\tilde{E}}}^{\overline{\tilde{E}}}(-1 / \varepsilon+0)-P_{\overline{\tilde{E}}}^{\overline{\tilde{E}}}(-1 / \varepsilon-0)\right\| \geqq 1-\beta(\delta)
\end{aligned}
$$

with

$$
\lim _{\delta \rightarrow 0} \beta(\delta)=0,
$$

so that replacing $\psi_{g}(-1 / \varepsilon-0)$ with $P_{-m}^{-}(-1 / \varepsilon+0) \psi_{g}(-1 / \varepsilon-0)$, the error is less than $\beta(\delta)$. Since $P_{-m}^{-}(-1 / \varepsilon+0)$ is contained in the absolutely continuous subspace of $H(-1 / \varepsilon+0)$, it follows that

$$
s-\lim _{\varepsilon \rightarrow 0} U_{\varepsilon, \lambda, \delta}(1 / \varepsilon,-1 / \varepsilon) P_{-m}^{-}(-1 / \varepsilon+0) \psi_{g}(-1 / \varepsilon-0)=0,
$$

wherefrom using also (3.12)

$$
\lim _{\varepsilon \rightarrow 0}\left\|P_{g}(1 / \varepsilon+0) \psi_{\varepsilon}(1 / \varepsilon)\right\| \leqq \beta(\delta) .
$$

Since $U_{\varepsilon, \lambda, \delta}(1 / \varepsilon,-1 / \varepsilon)$ commutes with $P_{-m}^{-}(-1 / \varepsilon+0)=P_{-m}^{-}(1 / \varepsilon-0)$,

$$
\left\|P_{-m}^{-}(1 / \varepsilon-0) \psi_{\varepsilon}(1 / \varepsilon)\right\| \geqq 1-\beta(\delta) .
$$

Now from (3.14), (3.15), Lemma 1 iii) and the definition of $\tilde{E}$,

$$
\begin{aligned}
\lim _{\varepsilon \rightarrow 0}\left\|P_{-m}^{-}(1 / \varepsilon+0) \psi_{\varepsilon}(1 / \varepsilon)\right\|= & \lim _{\varepsilon \rightarrow 0}\left\|\left(P_{\tilde{E}}^{\tilde{E}}(1 / \varepsilon+0)-P_{g}(1 / \varepsilon+0)\right) \psi_{\varepsilon}(1 / \varepsilon)\right\| \\
& \geqq 1-3 \beta(\delta) .
\end{aligned}
$$

Using again the adiabatic theorem for the interval $(1 / \varepsilon, 2 / \varepsilon)$ one obtains

$$
\lim _{\varepsilon \rightarrow 0}\left\|P_{0}^{-}(0) \psi_{\varepsilon}(2 / \varepsilon)\right\| \geqq 1-3 \beta(\delta) .
$$

Summing up

$$
\lim _{\varepsilon \rightarrow 0}\left\|P_{0}^{-}(0) U_{\varepsilon, \lambda, \delta}(2 / \varepsilon,-2 / \varepsilon) P_{0}^{+}(0) \psi_{\varepsilon}(-2 / \varepsilon)\right\| \geqq 1-3 \beta(\delta),
$$


which implies

$$
\lim _{\varepsilon \rightarrow 0}\left\|P_{0}^{-}(0) S_{\varepsilon, \lambda, \delta} P_{0}^{+}(0)\right\| \geqq 1-3 \beta(\delta),
$$

and the proof of the proposition is complete.

Remarks. 1. Consider a switching factor of the type considered in [1], i.e. $\{I\}=\phi$ and suppose $\varphi(s)=0$ for $|s|>1$, and $\frac{d}{d s} \varphi(s) \leqq 0$ for $s>0$ (these conditions are not essential and can be relaxed).

Let $\lambda>\lambda_{\text {cr }}$ and $-t(\varepsilon)<0$ be the time at which $E_{g}(t)$ reaches $-m$. One can try to repeat the "overcritical" part of the proof of Proposition 1, defining $\psi_{\varepsilon}(t)$ $=U_{\varepsilon, \lambda}(t,-t(\varepsilon)) \psi_{g}(-t(\varepsilon))$. The obstruction appears in proving (3.13), i.e.

$$
s-\lim _{\varepsilon \rightarrow 0} U_{\varepsilon, \lambda}(t(\varepsilon),-t(\varepsilon)) \psi_{g}(-t(\varepsilon))=0,
$$

and this is the technical difficulty mentioned in the Introduction.

2. Some time ago it was suggested that during heavy ion collisions an overcritical electric field is created for an instant, and then the spontaneous pair creation can be seen experimentally. Moreover a lot of calculations were performed (see [4-6] for details). The results of the experiment carried in Darmstadt [18] did not confirm the calculations. There are a lot of possible reasons: the calculations or/and the experiments are not accurate enough; the non-adiabatic effects are important, etc. It is not clear at present if the theory and the experiment will "converge" to a positive result or one has to look to a "cleaner" place for the spontaneous pair creation. As concerns the present note, the price paid for the fact that the results are rigorous is that the situation it describes is by far simpler than the actual complex situation in heavy ion collisions.

Acknowledgements. I would like to thank E. Seiler for discussions, and I am grateful to Prof. W. Zimmermann and E. Seiler for making possible my visit to Max-Planck-Institut für Physik und Astrophysik. The financial support of the Max-Planck-Institut für Physik und Astrophysik is gratefully acknowledged.

\section{References}

1. Nenciu, G.: On the adiabatic limit for Dirac particles in external fields. Commun. Math. Phys. 76, 117-128 (1980)

2. Nenciu, G.: Strong external fields, in Q.E.D. Rigorous results. Proceedings of the International Summer School in Heavy Ion Physics, Romania 1978

3. Bongaarts, P.J.M.: The electron-positron field, coupled to external electromagnetic potentials as an elementary $C^{*}$ algebra theory. Ann. Phys. 56, 108-139 (1970)

4. Rafelski, J., Fulcher, L.P., Klein, A.: Fermions and bosons interacting with arbitrarily strong external fields. Phys. Rep. 38 C, 227-361 (1978)

5. Brodsky, S.J., Mohr, P.K.: Q.E.D. in strong and supercritical fields in structure and collisions of ions and atoms. Sellin, I.A. (ed.). Berlin, Heidelberg, New York: Springer 1978

6. Reinhardt, J., Greiner, W.: Quantum electrodynamics of strong fields. Rep. Prog. Phys. 40, 219-295 (1977)

7. Scharf, G., Seipp, H.P.: Charged vacuum, spontaneous positron production and all that. Phys. Lett. 108 B, 196-198 (1982) 
8. Seipp, H.P.: On the $S$-operator for the external field problem of QED. Helv. Phys. Acta 55, 1-28 (1982)

9. Akhiezer, A.I., Berestetsky, V.B.: Quantum electrodynamics. New York: Interscience 1965

10. Abramowitz, M., Stegun, I.A.: Handbook of mathematical functions. Washington D.C.: U.S. Government Printing Office 1965

11. Hille, E.: Lectures on ordinary differential equations. Massachusetts: Addison Wesley 1969

12. Klaus, M., Scharf, G.: The regular external field problem in quantum electrodynamics. Helv. Phys. Acta 50, 779-802 (1978)

13. Nenciu, G., Scharf, G.: On regular external fields in quantum electrodynamics. Helv. Phys. Acta 51, 412-424 (1978)

14. Wightman, A.S.: Invariant wave equations; general theory and applications to the external field problem. In: Lecture Notes in Physics, Vol. 73. Berlin, Heidelberg, New York: Springer 1978

15. Simon, B.: Trace ideals and their applications. Cambridge: Cambridge University Press 1979

16. Kato, T.: On the adiabatic theorem of quantum mechanics. J. Phys. Soc. Jpn. 5, 435-439 (1950)

17. Nenciu, G.: Adiabatic theorem and spectral concentration. I. Arbitrary order spectral concentration for the Stark effect in atomic physics. Commun. Math. Phys. 82, 121-135(1981)

18. Cowan, T. et al.: Observation of correlated narrow-peak structures in positron and electron spectra from superheavy ion collisions systems. Phys. Rev. Lett. 56, 444-447 (1986)

Communicated by B. Simon

Received March 18, 1986; in revised form November 6, 1986 